Ann. Biol. anim. Bioch. Biophys., 1975, 15 (4), 785-788.

\title{
ÉTUDE DU RETENTISSEMENT DE LA CONTRACEPTION ORALE SUR DIVERS MÉTABOLISMES
}

\author{
G. LE LORIER, G. CATHELINEAU, J. P. GAUTRAY, \\ J. L. de GENNES, J. A. MARCEL et J. MENARD \\ Hôpital Saint-Antoine, Sevvice de Gynécologie-obstétrique, \\ 184, rue du faubourg Saint-Antoine, \\ 75012 Paris
}

RÉSUMÉ

Le but de ces recherches est l'étude du retentissement cle la contraception orale sur le métabolisme des lipides, le métabolisme des glucides, le système renine angiotensine, et enfin certains éléments de la coagulation sanguine, en particulier l'activité anti-thrombine progressive et les concentrations plasmatiques de trois antithrombines.

Ont été exclues de cette étude, les patientes diabétiques traitées par l'insuline ou les antidiabétiques oraux, les obèses, les malades présentant des antécédents de phlébite, d'embolie, de thrombose artérielle, ou encore d'hépatite de moins d'un an.

Sont également exclues les cardiopathies, les hypothyroïdies, les varices importantes et l'hypertension artérielle supérieure à I $6 / 9,5$.

Sur le plan local, après élimination d'une tumeur maligne pelvienne ou mammaire on a respecté les contrindications classiques de la contraception orale : fibrome, frottis suspects, adénome ou kyste du sein.

Les explorations imposéej à chacune des patientes par ce programme de recherches sont extrêmement contraignantes : les quantités de sang nécessaires pour pratiquer ces différents dosages sont relativement importantes. L'ensemble des facteurs étudiés ne peut l'être dans une seule séance, au cours d'un seul prélèvement. Par exemple, l'étude du métabolisme lipidique nécessitant deux prélèvements à 4 heures d'intervalle chez un sujet à jeun, après un repas à composition définie pris la veille dans la soirée, ne peut être réalisée le même jour que l'épreuve d'hyperglycémie par voie orale.

Au cours du cycle témoin, avant la prise d'westroprogestatifs, sont pratiquées 
successivement, en deux séances, une hyperglycémie provoquée puis une étude du métabolisme lipidique avec un prélèvement pour l'étude de l'hémostase et des facteurs rénine angiotensine.

Au bout d'un mois de traitement, une troisième séance de prélèvements étudie le métabolisme des lipides et les facteurs de l'hémostase.

Au bout du $3^{\mathrm{e}}$ mois de traitement, des prélèvements sont faits pour l'étude du système rénine angiotensine.

Au cours du $5^{\mathrm{e}}$ ou du $6^{\mathrm{c}}$ mois de traitement, on refait parallèlement des prélèvements pour l'étude du métabolisme glucidique, des prélèvements pour l'étude du métabolisme lipidique et du système rénine angiotensine.

Tous ces prélèvements sont répétés au cours du $\mathrm{r} 2^{\mathrm{e}}$ cycle.

Il faut noter aussi que chacun de ces prélèvements est réalisé selon un protocole précis, dans des conditions très strictes et qu'en particulier, pour l'étude du métabolisme glucidique et l'étude du métabolisme lipiđique, la patiente doit passer une matinée entière à l'hôpital. Malgré ces astreintes sévères, le recrutement de ces patientes a rencontré moins de difficultés que l'on aurait pu le craindre initialement.

La motivation de la plupart d'entre elles était la crainte d'un retentissement fâcheux des ostroprogestatifs sur leur santé, et le désir d'être rassurée à ce sujet dans toute la mesure du possible. Bien entendu il leur a été clairement exposé qu'une telle surveillance n'apportait de sécurité que dans des domaines très précis et ne pouvait répondre à toutes les interrogations que pouvait soulever le traitement œstroprogestatif. Un grand nombre de patientes a été également animé par le désir de participer à une enquête rigoureuse qui puisse être utile à la collectivité.

I'œstroprogestatif utilisé a été l'association de Norgestrel o,5 mg - F́thinylœstradiol o,05 mg spécialisé sous le nom de Stediril.

Ce produit a été choisi parce qu'il est apparu qu'à la date où cette étude a été mise en route, il était le plus fréquemment utilisé (il en est semble-t-il, de même à l'heure actuelle), et surtout parce qu'il se rangeait parmi les produits auxquels on attribuait le minimum d'effets secondaires. Or il était important, compte tenu des difficultés pratiques de l'étude, de prendre le minimum de risques d'abandon.

Cette étude doit être poursuivie sur roo femmes utilisant pour la première fois des ostroprogestatifs ou ayant interrompu le traitement depuis 3 mois au moins, mâis à i'heure actuelle seules 43 femmes âgées de 20 à 40 ans ont terminé la totalité des explorations pendant I2 cycles consécutifs. Ce sont les résultats obtenus chez ces 43 femmes qui seront commentés.

Indiquons cependant que $3^{8}$ autres patientes, ont par ailleurs terminé le $6^{\text {e }}$ cycle d'études, r 2 le $3^{c}$ cycle, I4 le premier cycle.

Sur l'ensemble des patientes ainsi recrutées, 27 abandons ont été enregistrés chiffre assurément important mais cependant plus faible que celui auquel on pouvait s'attendre, compte tenu de la sévérité des contraintes ainsi imposées.

Par ailleurs, nous ne pouvons faire encore état de certains résultats qui seront inclus dans 1'étude finale, car, par exemple la mesure de l'activité de la renine angiotensine plasmatique ne sera mesurée qu'en fin d'étude pour les roo patientes afin d'obtenir le maximum de précision dans les immuno-essais.

En ce qui concerne les perturbations de l'hémostase, on observe une baisse constante de l'antithrombine progressive et par ailleurs un abaissement significatif de l'alpha-globuline, dite antithrombine 3 . 
Fin ce qui concerne le substrat de la renine (angiotensinogène), les valeurs qui avant traitement varient de I 180 nanogrammes par millilitre, s'élèvent au bout d'un mois de 2200 à 5200 nanogrammes par millilitre. Au 6e mois les valeurs s'étagent de $375^{\circ}$ à 6 Ioo nanogrammes par millilitre, et se retrouvent an1 $12^{\mathrm{e}}$ mois entre 3600 et 5600 nanogrammes par millilitre. Au total, en ne tenant compte que des chiffres significatifs, on peut considérer qu'au bout d'un mois de traitement la valeur du substrat a été multiplié par 3 ou 4 et qu'elle est alors stabilisée.

Parallèlement 1'angiotensinogène mesuré chez des femmes recevant 25 microgrammes d'Éthinyl-œstradiol varie de 2500 à 3000 nanogrammes par millilitre. Les résultats cliniques rejoignent ainsi les résultats expérimentaux chez l'animal.

Comme l'augmentation du substrat de la renine s'accompagne d'une baisse de la sécrétion de renine, il faut disposer chez l'Homme d'une mesure de la renine indépendante du substrat. A cette fin a été étudiée une méthode de séparation de l'angiotensinogène et de la rénine qui devrait fournir la base d'une méthode de mesure de la concentration de renine.

Comme nous l'avons dit cette mesure sera appliquée en fin d'étude à l'ensemble des roo patientes.

Dans le domaine du métabolisme lipidique, on note au cours du I ${ }^{\text {er }}$ cycle une diminution non significative du cholestérol alors que les triglycérides amorcent une légère montée tout en restant dans les valeurs normales. Après le $6^{\mathrm{e}}$ et le $12^{e}$ cycles, le taux moyen du cholestérol augmente tandis que les triglycérides continuent à s'élever mais toujours dans les limites des valeurs normales. L'étude des pourcentages de variations individuelles montre que $82 \mathrm{p}$. roo des patientes au $6^{\mathrm{e}}$ cycle, 88 p. Ioo au $\mathrm{I}^{\mathrm{e}}$ cycle, ont une augmentation du taux de cholestérol inférieur à $30 \mathrm{p}$. IOO alors que seulement $6 \mathrm{I}$ p. Ioo au $6^{\mathrm{e}}$ cycle, $5^{2} \mathrm{p}$. Ioo au $\mathrm{I} 2^{\mathrm{e}}$ cycle ont pour les triglycérides une augmentation inférieure à $30 \mathrm{p}$. Ioo. Cela montre 1'inégalité des réponses d'une femme à l'autre vis-à-vis de l'action hyperlipidémiante de la pilule qui s'avère tout à fait insignifiante dans la majorité des cas, mais qui pour I2 p. Ioo des cas révèle une action hyperlipidémiante sans toutefois atteindre des chiffres pathologiques.

Cela confirme que pour atteindre des valeurs pathologiques sous pilule, il faut avoir un terrain prédisposant à un trouble de l'équilibre ou du métabolisme des lipides circulants.

C'est dans le domaine du métabolisme des glucides que l'étude en cours semble apporter les renseignements les plus intéressants. Pour cette étude, il a été convenu d'étudier sous hyperglycémie provoquée par voie orale, les modifications de la tolérance glucidique, de la sécrétion insulinique post-stimulative et de la sécrétion de l'hormone de croissance sur une période de 4 heures.

La tolérance glucidique est altérée par la prise de l'œestroprogestatif avec ou sans apparition d'une courbe de tolérance glucidique de type diabétique.

La sécrétion d'insuline est augmentée chez toutes les patientes. Il n'y a pas de corrélation entre les modifications et les données cliniques qui pourrait permettre d'attendre une détérioration de type diabétique de la courbe d'hyperglycémie provoquée par voie orale. Il n'y a pas non plus de corrélation entre les modifications de l'hyperglycémie provoquée par voie orale et l'augmentation des constituants lipidiques plasmatiques. 
Dans l'état actuel de l'étude, il semble que $25 \mathrm{p}$. Ioo des patientes s'individualisent par trois points :

I E Elles ont une courbe d'hyperglycémie anormale au bout de six mois.

$2^{\circ}$ Leur insulinémie est plus basse au départ, bien qu'elle se trouve augmentée, elle reste plus basse au bout de six mois que celle des femmes nornales.

$3^{\circ}$ Après six mois de traitement pour une même augnentation d'insulinémie, le contrôle de la glycémie n'est plus assuré.

Il est possible que la carence en vitamine $B_{6}$ soit responsable de la détérioration de la tolérance glucidique sous costroprogestatifs.

Dans l'avenir, l'étude du métabolisme du tryptophane et son évolution au cours du traitement ostroprogestatif, pourraient permettre de contrôler le métabolisme de la vitamine $B_{6}$ puisque, lorsqu'il existe une carence en vitamine $B_{6}$, cela entraîne une déviation du métabolisme du tryptophane vers l'acide xanthurénique, qui est capable de modifier le transport de l'insuline et de diminuer son action biologique in vitro sur le tissu musculaire et adipeux.

Collogue D. G. R. S. T., Biologie de la Procréation, Paris, 7-8 Mars 1975.

\section{REMERCIEMENTS}

Ce travail a bénéficié de l'aide de la 1). G. R. S. T., contrat n0 $72-70788$.

\section{SUMMARY}

\section{EFFECT OF ORAL CONTRACEPTION ON SOME METABOLISMS}

The evolution of different biological factors is studied for a one year follow-up of estroprogestative treatment.

During hemostasis there is constant fall of progressive antithrombin and significant lowering of the 3 antithrombin. On the other hand, angiotensinogen level is 3 or 4 times higher as early as the end of the first month of treatment.

Results on lipid metabolism vary greatly from one woman patient to another. In many, the hyperlipemic action of estroprogestatives is quite trivial, but in $\mathrm{r} 2 \mathrm{p}$. Ioo of the women the action is manifest without reaching pathological levels. It is possible that pathological levels are only reached when the subjects are predisposed.

Modification of glucose tolerance, post-stimulative insulin secretion and growth hormone secretion are studied during four hours after an oral glucose test. There is impairment of glucose tolerance, with or without appearance of a diabetic glucose tolerance curve and a regular rise of insulin secretion.

At the beginning of the experiment, 25 p. roo of the patients have a lower insulin level, which does not rise sufficiently to show normal glucose tolerance test six months later. 